\title{
EXTENDED HALOS IN THE CLUSTER ABELL-426
}

\author{
Janos Kelemen \\ Konkoly Observatory \\ Budapest \\ Hungary
}

\author{
Claus Mollenhoff \\ Landessternwarte Heidelberg-Königstuhl \\ FRG
}

\begin{abstract}
During 1987 and 1988, we obtained 12 long-exposure Schmidt plates of the cluster Abell-426 at the Konkoly Observatory. The pictures were digitized with a microdensitometer and added with a computer. The resulting blue images clearly show extended envelopes around the bright cluster members.
\end{abstract}

The Abell-426 (Perseus cluster) is a chainlike L-type cluster that shows luminous X-ray radiation and has a high radio luminosity. These two features suggest that this cluster contains a significant amount of intracluster gas. The NGC 1275, which lies very near to the core of this cluster, is one of the most interesting extragalactic objects.

Due to these very interesting features, a common program was developed between the Landessternwarte Heidelberg-Königstuhl in the Federal Republic of Germany and the Konkoly Observatory in Budapest, Hungary. Many long-exposure plates were obtained at the mountain station of the Konkoly Observatory with a 60/90 cm Schmidt telescope during the period 1987-1988. The photomaterial was KODAK IIIaJ and the plates were hypered in dry nitrogen. The exposures were taken without any filter and the duration of the exposures were between 1.5 and 3 hours.

After visual investigation, we chose a subset of 12 of the highest-quality plates for further analysis. The plates selected are detailed in Table 1.

TABLE 1. Photo Material Employed

\begin{tabular}{cclccc}
\hline \hline No. & Date & exp. & No. & Date & exp. \\
\hline S11550 & $28 / 29.10 .87$ & 1.5 hour & S11926 & $04 / 05.11 .88$ & 2 hour \\
S11551 & $28 / 29.10 .87$ & 1.5 hour & S11927 & $04 / 05.11 .88$ & 2 hour \\
S11553 & $29 / 30.10 .87$ & 1.5 hour & S11928 & $05 / 06.11 .88$ & 2 hour \\
S11600 & $15 / 16.01 .88$ & 2 hour & S11930 & $05 / 06.11 .88$ & 2 hour \\
S11856 & $08 / 09.10 .88$ & 2 hour & S11931 & $05 / 06.11 .88$ & 2 hour \\
S11857 & $08 / 09.10 .88$ & 2 hour & S11938 & $10 / 11.11 .88$ & 2 hour \\
\hline
\end{tabular}

We digitized the plates with the aid of a PDS microdensitometer. Two kinds of digitalization were made, one with $1500 \times 1500$ steps and $10 \times 10$ micron pixel size and the other with $1000 \times 1000$ steps and $50 \times 50$ micron pixel size. In the digitalization, we did not use overlapping pixels, so the measured fields were $15 \times 15 \mathrm{~mm}$ and $50 \times 50 \mathrm{~mm}$, respectively.

The digitalized pictures were co-added in a computer system equipped with the MIDAS image processing system. The estimated magnitude limit for stars is about 22 magnitude. Figure 1 shows the result of the digital additions. These results show that the brightest members in the cluster core have extended optical envelopes. The same is true for the outer region of NGC 1275.

Acknowledgments-This project was financially supported by SFB 328. 


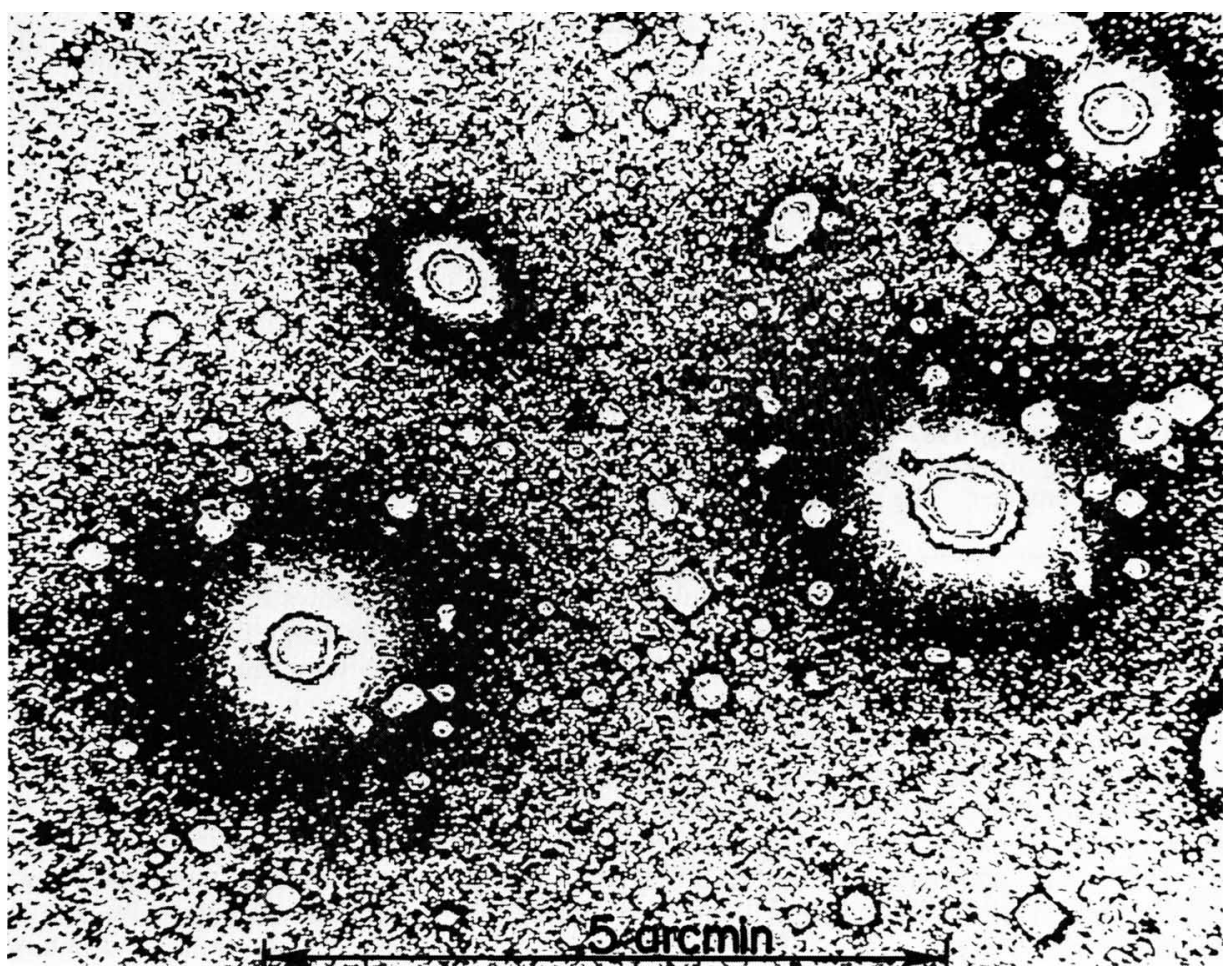

Figure 1. The core "quartet" of the Abell-426. This picture is the $500 \times 500$ pixel wide central region of the $(1500 \times 1500$ pixel) original version. Orientation: North is at top of page, east to the right. Scale: 5 arcmin $=110 \mathrm{~mm}$. Extended envelopes are clearly shown, even around NGC 1275 (middle right). 